\title{
Anaphylactic shock-induced ischemic proctocolitis following bee stings: first case report
}
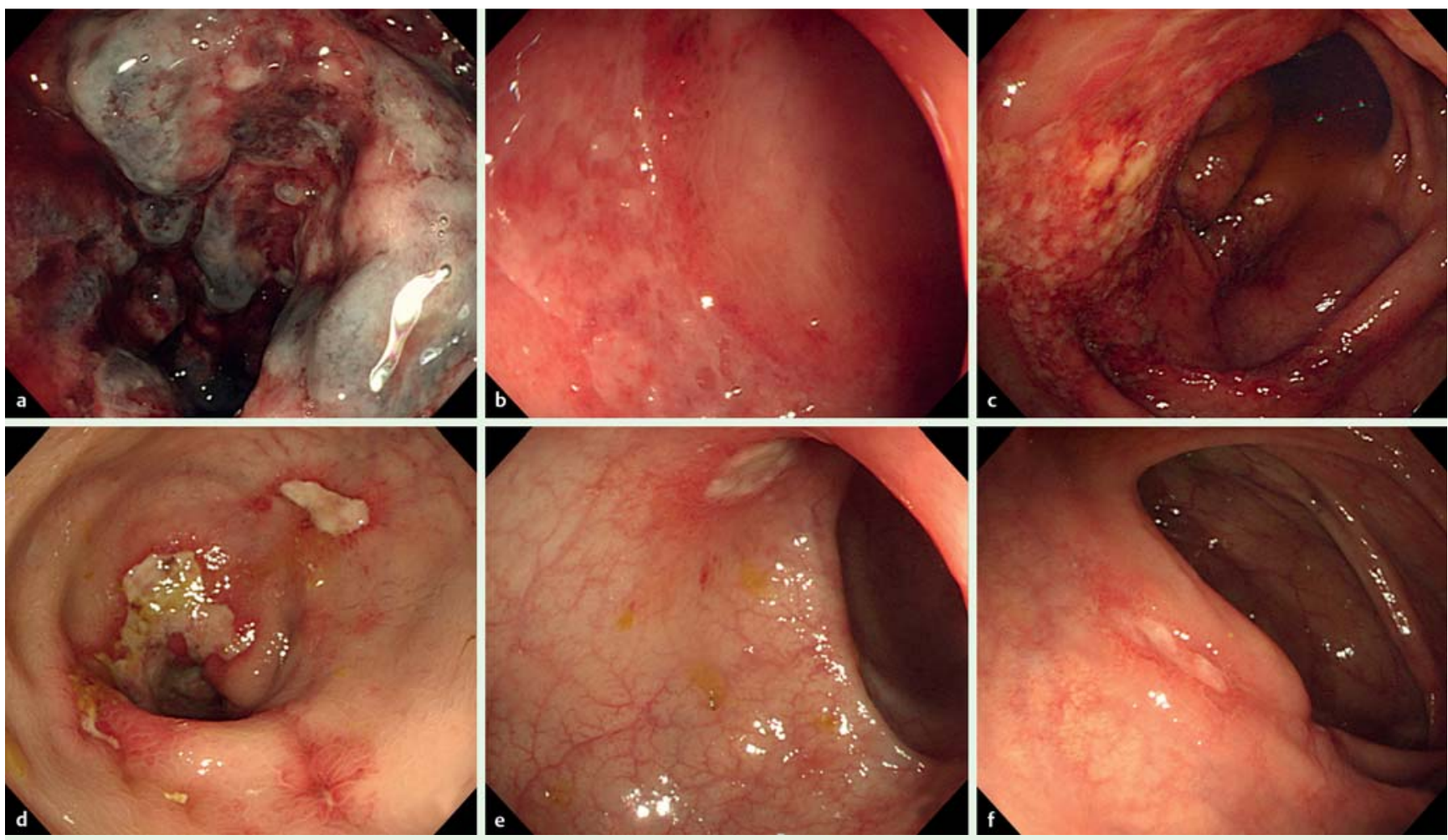

Fig. 1 Colonoscopic findings. a Severely edematous and hemorrhagic mucosa with narrowing of the rectal lumen. $\mathbf{b}$ The proximal sigmoid colon representing the transitional zone leading on to a normal mucosal appearance. $\mathbf{c}$ Cecal examination showing a patchy erythematous surface in the region of the ileocecal valve, covered with exudates. $\mathbf{d}$ - $\mathbf{f}$ Significant improvement of the internal findings shown in a-c, respectively, on follow-up colonoscopy carried out 14 days after admission.

A 41-year-old male patient was referred for hematochezia following resuscitation from a hypotensive shock. The history revealed that while working outdoors, the patient had been attacked by a swarm of bees and received multiple stings, rendering him unconscious. Following initial recovery at the local hospital, he developed hematochezia and was referred to us. On examination at admission, the patient was alert and not pale, and his vital signs were stable. He denied any previous allergic history related to drugs or other substances, and he was not taking any medication. There was no notable family history. Pain and tenderness were elicited in the periumbilical region but without rebound tenderness. Digital rectal examination revealed bloody and mucoid discharge. The initial laboratory results showed leukocytosis and mild elevation of serum aminotransferases without anemia. Abdominopelvic computed tomography (CT) disclosed edematous wall thickening in the rectosigmoid and cecal regions. Electrocardiography did not show any sign of cardiac arrhythmia. On sigmoidoscopy, the rectal mucosa was hemorrhagic and greatly swollen with narrowing of the lumen, whitish exudates, and intermittent, deep ulcerations ( Fig. 1a). This presentation persisted as the scope was advanced and then relatively normal mucosa was seen at $25 \mathrm{~cm}$ from the anal verge with a sharply distinct margin ( Fig. 1 b). Total colonoscopy carried out the next day revealed cecal pathology with similar findings ( Fig. 1 c). Following administration of empirical antibiotics, bowel rest, and copious intravenous hydration, the abdominal pain subsided and the hematochezia ceased. The patient resumed his normal diet 11 days after admission. Pathologic examination verified the diagnosis of ischemic colitis. Followup total colonoscopy before discharge revealed much improvement ( $\bullet$ Fig. $1 \mathbf{d}-\mathbf{f}$ ), and a later endoscopic review examina- tion revealed minimal changes in the colonic mucosa with no evidence of stricture or other deformities.

Ischemic colitis is the most common manifestation of mesenteric ischemia [1], and a few cases of acute ischemic colitis of unusual etiology have been reported [25]. Our patient's young age and otherwise apparently healthy constitution with no notable medical history are suggestive of low probability of atherosclerotic change and susceptibility to any kind of bowel ischemia. Our patient therefore exemplifies a rare instance of anaphylactic shock caused by bee stings leading to ischemic colitis, and is the only case of its kind to be reported to date.

\section{Competing interests: None}

Endoscopy_UCTN_Code_CCL_1AD_2AJ 
S. Park, H. J. Chun, B. Keum, Y. S. Seo, Y. S. Kim, Y. T. Jeen, H. S. Lee, S. H. Um, C. D. Kim, H. S. Ryu Department of Internal Medicine, Institute of Digestive Disease and Nutrition, Korea University College of Medicine, Seoul, Korea

\section{References}

1 Paterno F, Longo $W$. The etiology and pathogenesis of vascular disorders of the intestine. Radiol Clin North Am 2008; 46: 877 885

2 Prez-Carral C, Carreira J, Vidal C. Acute ischaemic colitis due to hypotension and amoxicillin allergy. Postgrad Med J 2004; 80: $298-299$

3 Travis S, Davies DR, Creamer B. Acute colorectal ischaemia after anaphylactoid shock. Gut 1991; 32: $443-446$

4 Shirai T, Mori M, Uotani T, Chida K. Gastrointestinal disorders in anaphylaxis. Intern Med 2007; 46: 315-316

5 Rivera RA. Gastroscopic observations during anaphylaxis in a human. Gastrointest Endosc 1972; 19: 21 - 22

\section{Bibliography}

DOI $10.1055 / \mathrm{s}-0029-1244164$

Endoscopy 2010; 42: E153 -E154

(c) Georg Thieme Verlag KG Stuttgart · New York . ISSN 0013-726X

\section{Corresponding author}

\section{H. J. Chun, MD, PhD, AGAF}

Department of Internal Medicine Korea University Medical Center

126-1, 5-Ga, Anam-Dong

Seongbuk-Gu

Seoul 136-705

Korea

Fax: +82-2-9531943

drchunhj@chol.com 